\title{
Virus-Like Particles: Applications in Reverse Vaccinology
}

\author{
Agustin De Ganzó ${ }^{1, *}$, Mercedes Pastorini ${ }^{1}$, Cristina Borio ${ }^{1}$, Mario Lozano ${ }^{1}$, and Sandra Goñi ${ }^{1}$ \\ Area de Virosis Emergentes y Zoonóticas (AVEZ), Laboratorio de Ingeniería Genética y Biología Celular y \\ Molecular (LIGBCM), Departamento de Ciencia y Tecnología (DCyT), Universidad Nacional de Quilmes \\ (UNQ), Roque Sáenz Peña 352, B1876BXD, Bernal, Buenos Aires, Argentina; agus@ad.unc.edu; \\ mercedes.pastorini@alu.unq.edu.ar; cborio@unq.edu.ar; mario.lozano@unq.edu.ar; sandra.goni@unq.edu.ar \\ * Correspondence: agus@ad.unc.edu; Tel.: (optional; include country code; if there are multiple \\ corresponding authors, add author initials) +1-919-808-6503 (F.L.)
}

\begin{abstract}
Vaccination has been one of the most successful and the most significant scientific advances in human health and life expectancy all around the globe. The World Health Organization considers that immunization should be recognized as the main component of human health right, due to the fact that vaccination prevents 2.5 million deaths annually (World Health Organization, 2011). The most successful vaccines have been developed using conventional methods that follow the paradigm established by Pasteur: "to isolate, inactivate and inject" the pathogen microorganism and mimic a natural infection. Recently, metagenomics have played an important role in the discovery of new immunogens for vaccine design and the selection of antigens based on genomic information. The main approach that has used this strategy has been called "reverse vaccinology". This promising and arising field allows the screening of the entire potential antigenic repertoire of an organism using predictive bioinformatic tools. Once the antigenic protein or proteins have been selected, they are expressed and purified using molecular cloning and in vitro expression techniques. Following the in vitro production step, they are probed in animal models to evaluate the in vivo protective strength of the immune response. The main aim of this in vivo approach is to evaluate the ability of the immune response to eliminate or neutralize the pathogen at the time of infection. Those antigens capable of generate a specific immune response with neutralizing activity for natural infections are the best candidate vaccines. In this review we summarize the evolution of vaccinology since its inception, with special emphasis on the development of VLPs as vaccine platforms and their future in preventive medicine and we introduce a new recombinant platform for antigen presentation based on Junin virus VLPs (JUNV-VLPs).
\end{abstract}

Keywords: VLPs; vaccines; reverse vaccinology; JUNV; vaccinology

\section{Introduction}

Since the discovery of the smallpox vaccine in 1796 and the origin of classic or conventional vaccinology, there have been numerous advances against infectious diseases. In the past, vaccines and therapeutic treatments were developed without the basic knowledge of the pathogenesis of the infectious agents. Thus, classical vaccination has been built with minimal knowledge of the complex immunological mechanisms by which vaccines induce protective immunity [2], [3].

Genomics has radically changed the identification of vaccine candidates. As a result of several technology revolutions, it is possible to identify new vaccine antigens using genomic, transcriptomic and proteomic analysis on a large scale. In addition, the advance in Bioinformatics have introduced 
new methods for the design of antigens, and the analysis of its induced immune response [4]. Genomics-based technologies have also revealed novel virulence factors for various pathogens.

\subsection{Vaccinology: Pasteur's Principles and Vaccine Development}

The success of a certain vaccine depends entirely on the activation of the protective immune components of the organism itself. Live attenuated or inactivated vaccines have been successful because they mimic the type of protective immunity induced in people who has survived a natural infection.

Most of the vaccines used today have been developed using conventional methods that follow the paradigm established by Pasteur: to "isolate, inactivate and inject" the microorganism that causes the disease and, thus, imitate a natural infection. In this strategy, it is important to preserve the following factors: 1) reducing or eliminating the infectivity of the pathogen (attenuation and/or inactivation), 2) inhibiting its ability to replicate, 3) preserving their immunogenicity.

Pasteur applied his principles to viruses (rabies viruses isolated from rabbits), and bacteria (heat-inactivated anthrax bacilli) with great results. However, these approaches have been successful so far for those pathogens with invariant antigens, and those that after a single infection can induce an immune memory capable of preventing subsequent re-infections, such as smallpox, yellow fever, poliomyelitis, rabies, Mumps, measles, rubella, varicella, herpes zoster, hepatitis A, and Japanese encephalitis [5]. In addition, attenuated inactivated or live vaccines have also been developed against some infectious bacterial diseases with different successfulness; the Calmette-Guerin Bacillus (live attenuated Mycobacterium bovis) is possibly the most widely used vaccine and is currently the only available against tuberculosis [6].

However, classical or conventional vaccination has proven to be inadequate in the development of vaccines against pathogens with different characteristics: a) pathogens antigenically diverse; $b$ ) pathogens that cannot be grown in vitro; c) pathogens lacking adequate animal models of infection; and d) pathogens that stimulate cell-dependent immune response ( $T$ cells).

These difficulties were solved with the development of subunit vaccines based on Pasteur's strategy: essential components of the pathogen, usually virulence determinant subunits, were isolated and inactivated to be used as vaccines. This approach was first applied for diphtheria and tetanus. At the present, subunit vaccines have grown significantly and their ability to generate long-term immune responses and immune memory has been optimized. These types of vaccines are safe and effective, and multivalent formulations have been developed, for example, to provide protection against numerous pneumococcal serotypes and meningococcal serogroups in a single shot.

Although the virtual disappearance of diseases such as diphtheria, tetanus, whooping cough and Haemophilus influenzae type $b$ are references for the success of vaccines, there are still many diseases where vaccination strategies have not been successful so far. This is due not only to the hypervariability of their antigens but also to an incomplete understanding of pathogenicity mechanisms and cell-mediated immune responses [7]. A clear example is HIV (human immunodeficiency virus), an infection that stimulate a virtual adaptive humoral immunity, and therefore, vaccines must induce cellular immunity with low or lack of protective potential over time [8], [9].

Recombinant techniques to develop subunit vaccines and genetic inactivation by targeted mutagenesis, has made possible to generate attenuated live vaccines and safer subunit vaccines through the design in silico. This technology has also generated a wide range of new platforms vaccines and ways of administration. Recombinant antigens from a subunit vaccine are usually better tolerated than live attenuated or inactivated pathogens or purified "natural" subunits for vaccination. This is because they are produced in cell cultures from the natural host and their proteins exhibit identical posttranslational modifications to those present in wild type infectious virions lacking other viral immunogens that might generate inflammation or unspecific immune response. However, the main limitation is the low immunogenicity that might result in the addition of an adjuvant to generate a protective immune response [10].

\subsection{Reverse vaccinology}


Genome-based antigen selection has played an important role in the discovery of new immunogens for vaccine design in the past years. The use of these approaches has been called "reverse vaccination" and has enabled the screening of the whole potential antigenic repertoire of an organism through its genome using bioinformatic predictive tools [11].

Reverse vaccinology involves the in-silico prediction of proteins with immunogenic characteristics from the genome of a pathogen organism using bioinformatic methods. The predicted proteins are expressed recombinantly as surface antigens or secreted in monomeric or oligomeric forms to be purified using in-vitro molecular cloning methods and evaluated in animal models to test their ability to generate a protective immune response. Those antigens capable of generating a specific immune response with neutralizing activity for future natural infections are the best candidate vaccines.

This approach has been applied to a wide range of pathogens and has provided a long list of antigens whose functionality was unknown [12]. Further studies on the function of each antigen also allow a better understanding of the biology of pathogens. One example is the use of protective antigens implicated in bacterial adhesion, as there may be important implications for providing immunity by reducing colonization and circulation of the pathogen. In fact, functional studies of many protective antigens have subsequently revealed that they are new adhesins involved in the colonization of the pathogen[13], [14].

Although a vaccine antigen does not necessarily have to be a virulence factor; focusing on proteins with a crucial role in pathogenesis or survival is a useful selection criterion since the antibodies induced against this type of antigens can block their function and result in the general neutralization of the pathogen.

The first pathogen in which reverse vaccination was used was Neisseria meningitidis serogroup B [15]. Pizza and colleagues identified 29 new antigens that could induce a bactericidal humoral response against the pathogen in vitro. Studies performed on three of the major antigens included in the MenB vaccine (Neisseria heparin binding antigen, NHBA, factor $\mathrm{H}$ binding protein, $\mathrm{fHbp}$, and adhesin $\mathrm{A}, \mathrm{NadA}$ ) have subsequently been reported as important factors of virulence in meningococcal infection [13]. Several advances have been made since this first application of reverse vaccination. In 2005, Maione and colleagues [16] applied a "multi-genome or pan-genome reverse vaccinology" approach to streptococcus group B to identify antigens from the complete gene pool of the species rather than a single organism; more recently the "subtractive reverse vaccination" approach has been implemented to identify antigens present in non-edible pathogenic strains of Escherichia coli [17].

\subsubsection{Rational design of vaccines}

Currently, the main application of reverse vaccination is the rational design of candidate vaccines. The rational design follows a stepwise approach (Figure 1): 1) identification of a target antigen, 2) selection of a vaccine platform, 3) optimization of factors for gene expression, 4) production and characterization of the candidate vaccine, and finally 5) analysis of the biosafety, immunogenicity and efficacy cellular models in vitro and in animal models [18].

In virology, recent advances in molecular biology and bioinformatics have contributed significantly to the understanding of the structure, replication, attenuation and pathogenicity determinants. The knowledge of these aspects is crucial to identify target proteins [18]. Computational vaccinology is the field that focuses on the process of antigenic identification in a predictive manner. Most of the bioinformatics tools focus on the presentation and processing of antigens and try to aim the response towards $\mathrm{T}$ and $\mathrm{B}$ cells. Although the mapping and identification of highly immunogenic epitopes on target antigens is important, several additional steps should be considered when designing new vaccines rationally. 


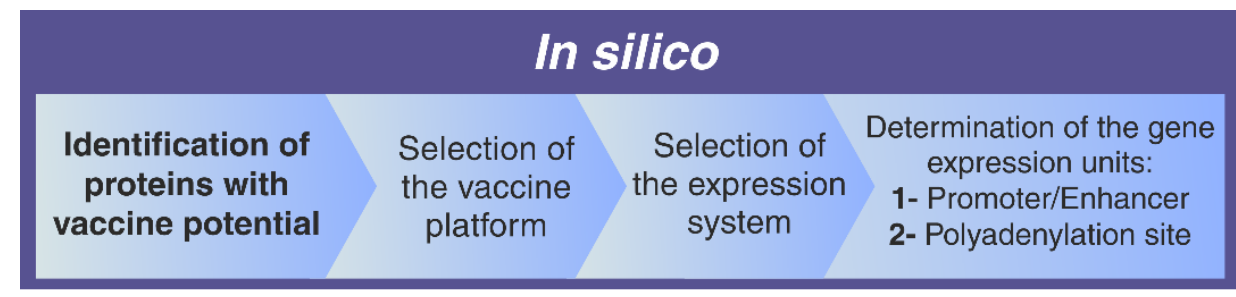

\section{In vitro}

\section{Expression and analysis}

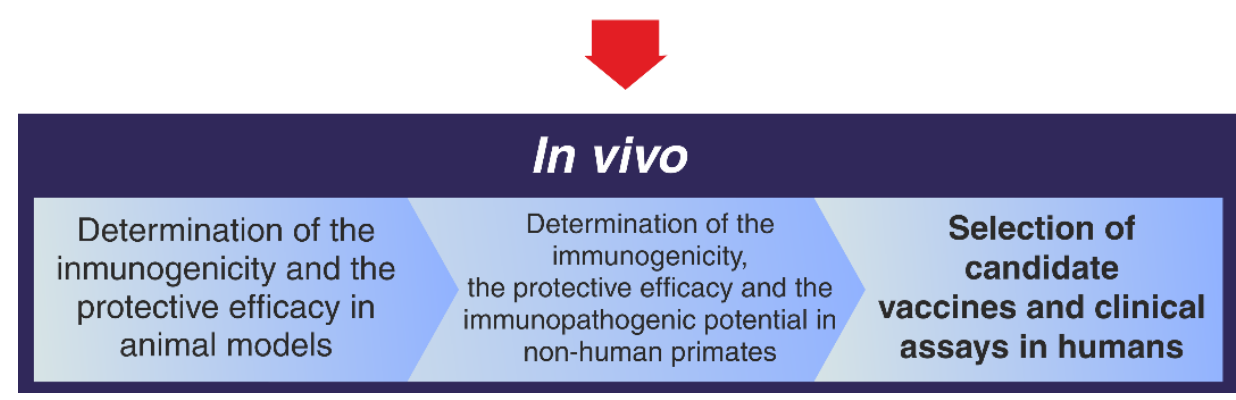

Figure 1. Rational design of candidate vaccines approach. The rational design of candidate vaccines can be easily summarized in the three conventional stages: during the in silico stage every identification and selection is made using bioinformatic tools; the in vitro stage consists of the molecular cloning of the selected sequences into the expression vector, the protein expression and its analysis; finally, the results obtained in the in vivo stage will determine the use of the selected candidate.

\subsection{Structural vaccinology and virus-like particles (VLPs) as vaccine platforms}

Structural vaccinology is an emerging discipline that aims to identify, design and evaluate antigens with vaccine potential based on studies of structural biology, computational biology, immunology and serology [19], [20]. This emerging field also uses transcriptomics and proteomics tools that can inspect the set of antigens expressed by a pathogen under specific conditions, examining the mRNA and the proteins of an organism, respectively. Moreover, the analysis can be focused only on one subset of proteins: those exposed on the surface (surface proteome) or those functionally important for infection (functional genomics) [4]. There are also new fields of study focused on elucidating the set of antigens that interact with the host's immune system and the mechanisms involved in these interactions (immunomics) as well as the way in which the immune system responds to a vaccine (vaccinomics). While each of these approaches has limitations, they have all emerged as powerful tools in the development of vaccines and are directly linked to structural vaccinology.

Although the technology improvement and the fact that modern vaccines contain safer antigens, the levels of protective immunity induced are lower when compared to nineteenth and early twentieth century vaccines.

One approach that attempts to overcome this disadvantage is the use of virus-like particles (VLPs) [21]. VLPs have the same repeatability, geometry, size and morphology of natural virions and lack the original viral genome. These VLPs can be composed of single or multiple antigens, in some cases anchored in a lipid bilayer and are generated by the self-assembling activity of some structural proteins of different virus. VLPs are used as platforms for the 
presentation of immunogenic epitopes: the pIII protein of filamentous phage f1 [22], the Ty component of Saccharomyces cerevisiae [23], the surface or layer proteins of the bluetongue virus (BTV) [24], the human parvovirus B19 [25], the tobacco mosaic virus (TMV) [26] and the human papilloma virus (HPV) [27] are some examples.

The rational design of vaccines combined with structural vaccinology and the emerging of self-assembling VLPs are currently the most promising field to develop generic vaccine platforms and there are currently available several VLPs-based vaccines [28].

\subsubsection{VLPs: impact on vaccinology}

VLPs are self-assembling supramolecular associations that do not carry any genetic information, are non-replicative and can be produced in heterologous expression systems. VLPs are biosafe given the lack of genetic information and that they preserve the immunological characteristics of the original virus that induce immune response [29] Furthermore, due to the fact that many antigens can be exposed on the surface of VLPs, they are powerful tools in immunology and vaccinology since they can be used as vaccine platforms and therapeutic delivery systems [30][33]. It has been reported that VLPs stimulate the immune system completely, they: a) stimulate innate immunity through Toll-like receptors (TLRs) and pattern recognition receptors (PRRs) due to the expression of multivalent and repetitive structures; b) induce a strong humoral response and IgM in T-cells independently; and c) increase affinity by enhancing the maturation of APCs (Antigen Presenting Cells), and processing and presentation by the APCs through the route of the MHC-I and MHC-II (Major Histocompatibility Complex class I and class II) due to their particulate nature [34].

\subsubsection{Structure of the VLPs}

VLPs have been generated from a large number of anthroponotic and zoonotic viruses [32], [35]-[37]. They are composed of one or more structural or capsid proteins capable of self-assembly into virion-like morphological structures. Compared with complete virions, they do not replicate or infect host cells due to the absence of genetic material. In addition, it has been reported that they can be used as vaccines without artificial adjuvants [38], [39].

The structural proteins responsible for the generation of VLPs vary depending on the biology of the virus: a) simple viral capsids with one or two major structural proteins (Parvovirus, HPV, Circovirus, Calcivirus, hepatitis E virus (HEV) and Poliomavirus); b) complex viral capsids with several layers of viral proteins encoded by different mRNAs or generated from a single polypeptide (Rotavirus, Picornavirus), or c) enveloped viral capsids derived from the host cell membrane that may include surface glycoprotein spikes (Influenza, HIV, hepatitis C (HCV) and arenavirus [40].

Recently, there has been described viral nanoparticles (VNPs) and VLPs derived from plant viruses [35].

\subsubsection{Processing of VLPs by the immune system}

VLPs are commonly more immunogenic than subunit immunogens or recombinant proteins and are capable of stimulating both the humoral and cellular immune responses [41]. VLPs can be inoculated subcutaneously, intraperitoneally or intramuscularly. Their small diameter facilitates the entry into the lymphatic system and drains directly to local nodes. Once in the lymph node, the VLPs are processed mainly by dendritic cells (DCs); the capture mediated by the DCs is improved by the shape and size of the VLPs, identical to those of the infectious 
virions. Once processed, they stimulate the activation and proliferation of T-CD4+ cells through the MHC II and the MHC I pathways and induce innate immunity.

\subsection{Induction of innate immunity}

In some cases, VLPs do not require the use of adjuvants to achieve efficient immune stimulation. The self-adjuvant effects are inherent to their adequate size; this facilitates their uptake by the DCs, their subsequent processing and presentation by MHC class II, and the promotion of the maturation and migration of DCs, essential for the innate immune response [42], [43]. VLPs can also be captured and processed through the MHC class I pathway (cross-presentation) to achieve the activation of T-CD8 + cells, essential for the elimination of intracellular pathogens. The ability of VLPs to aim the DCs is an important advantage of VLP vaccines since it is now understood that these cells are essential to initiate both innate and adaptive immune responses.

On the other hand, the VLPs that retain their receptor binding regions are able to enter cells through their regular cellular receptor and are captured afterward by APCs as exogenous antigens. It has been shown that receptor-mediated uptake occurs in paramyxoviruses and in HIV [44], [45]. In the case of the stimulation of DCs to produce cytokines, such as interferon- $\alpha$ / $\beta$, it does not require the virus replication, but an intact envelope of an inactivated virus [46] or a non-enveloped VLP, as in the HPV-VLPs [47]. In this context, VLPs may have very important advantages over attenuated viruses for immune activation, because several viruses that replicate in DCs impede their activation and maturation through the expression of particular viral proteins. [48].

\subsection{Induction of adaptive immunity}

In order to induce humoral immunity in higher antibody titers, the target antigens could be exposed on the surface of the VLPs at high densities. VLPs imitate the structure of the native virions and have a spatial structure that allows the correct detection of the conformational epitopes, and consequently they increase the production of neutralizing antibodies. This is especially important for surface proteins, where enveloped VLPs allow proteins to appear in their natural state as membrane-anchored proteins, rather than soluble ectodomains [49].

Hence, VLPs can offer a safe and effective approach for the induction of antibodies by surface proteins where the soluble forms have not been effective [35]. Some VLPs have also the ability to present multiple proteins to the immune system. For example, the successful hepatitis B virus (HBV) vaccine has been optimized by including two other envelope proteins in the HBsAg particle, thus mimicking the authentic virion envelope that contains these three proteins. HBV vaccines that include these additional envelope proteins stimulate the production of antibodies differently and more specifically due to the provision of additional $\mathrm{T}$ cell epitopes that promote a more complete $\alpha$-HBsAg response and improve the rate of seroprotection [50].

Another important example is HIV. Great efforts have been made to recreate the virion envelope that allows efficient induction of neutralizing antibodies. VLPs were produced in the form of synthetic proteoliposomes in solid microspherical beads in order to mimic the lipid membrane for the presentation of the HIV glycoprotein GP160 [51], as well as VLPs mimicking the HIV particle containing the envelope proteins associated with its matrix protein Gag [52]. This, in addition to the advances made by reverse vaccinology, has helped the understanding that the key domains associated with viral entry and conserved regions probably do not experience antigenic variation despite neutralizing immunological pressure. Recently, the research has focused on the presentation of highly conserved and neutralizing epitopes of the envelope protein GP41 of HIV-1 in a pre-fusion conformation, which is only briefly exposed during viral entry [53]. 


\subsubsection{VLPs as vaccine platforms}

VLPs can be used as potential vaccines against the original viruses as well as displaying platforms for foreign individual epitopes. This can be done by gene fusion and subsequent expression of the resulting recombinant protein or by chemical conjugation between the target antigen and the structural protein that generates the VLPs, offering new perspectives in the development of vaccines [21].

One of the best achievements of VLPs as vaccine platforms is the vaccine against malaria, a chimeric and recombinant VLP (rVLPs) made of the circumsporozoite protein of Plasmodium falciparum and the surface antigen of hepatitis B virus. This candidate vaccine, called RTS-S, has reached phase III clinical trials and induces a protection of approximately 30-50\% [54]. Despite this moderate efficacy, which can be explained by the low expression of the antigen on the surface of the VLPs, the RTS-S is the first vaccine that shows significant protection against malaria, where other strategies such as synthetic peptides and viral vectors have failed so far.

Likewise, other platforms have been used to display foreign antigens. Using simple structures made up of self-assembling proteins such as the HBcore protein, the envelope protein of the alfalfa mosaic virus, the HPV L1 protein or the bacteriophage $\mathrm{Qb}$, has been shown to effectively stimulate specific immune responses against displayed antigens [55]-[57]. The structural proteins are crucial because they influence the overall immunogenicity. These studies demonstrate the great potential of the rVLPs since many of them have already reached the phase of clinical trial [36]. The rVLPs derived from enveloped viruses also offer an encouraging panorama to integrate target antigens on their surface and anchored in their membrane.

Another viable strategy for antigenic displaying is pseudotyping, in which pseudo-viral particles are decorated with foreign antigens using genetic engineering. It is important to highlight that pseudoviruses, unlike VLPs, are complete viruses that carry foreign proteins that are not encoded in their genome, providing extra functions during infection that will not be present in the viral progeny. This approach has two important advantages: a) displaying the foreign antigens in its native conformation and, b) optimization in the uptake by the APCs. Retroviral nuclei have been shown to be adaptable for this approach. retroVLPs based on simple oncoretroviruses (retrovirus-like particles) have been developed using the retrovirus of murine leukemia (XMRV, Xenotropic murine leukemia virus). The expression of the matrix protein Gag is sufficient to generate enveloped pleomorphic particles of 80-120 nm, which can be pseudotyped with different proteins without weakening its functionality, such as glycoproteins of the lymphocyte choriomeningitis virus (LCMV), the virus of splenic necrosis (SNV), the vesicular stomatitis virus (VSV), the HCV, the virus of the Yellow fever (YFV), West Nile virus (WNV), Influenza virus, HIV or cytomegalovirus (CMV) [58], [59], without weakening its functionality.

\subsection{Current developments in Junín virus' virus-like particles (JUNV-VLPs)}

In our laboratory we generated JUNV-VLPs in HEK-293T cells and we were able to evaluate the immune response in mice [60], [61]. The plasmid vector pZ-GFP includes the sequences of the JUNV matrix Z protein and GFP. These VLPs were able to induce humoral response in mice and we are currently analyzing the qualitative induction of the cellular response. Based on these results and on the interaction between the $\mathrm{Z}$ protein and the other proteins of the virion, we designed and developed a plasmid that allows the heterologous expression of foreign antigens anchored to the JUNV-VLPs membrane. The design includes the minimum genomic elements of anchoring and membrane targeting of the JUNV glycoprotein complex GP-C and the cytosolic domain that interacts with $Z$ to ensure their incorporation into the VLPs [62]. This vector was named pResAg, for presenting antigens plasmid (Figure 2). Although we have been 
able to clone different viral antigens in pResAg (the ectodomain of the measles virus hemagglutinin and the Saint Louis virus NS1 protein) and we have detected their correct expression with specific antibodies [62], the validation of the model is being carried out with the eGFP indicator protein due to its obvious fluorescent advantages and for its properties of conferring stability to the heterologous proteins that include its sequence. These trials, together with the inoculation of laboratory animals and the evaluation of the immune response generated in vivo, will allow us to evaluate the ability of pResAg to be used as a future vaccine platform.

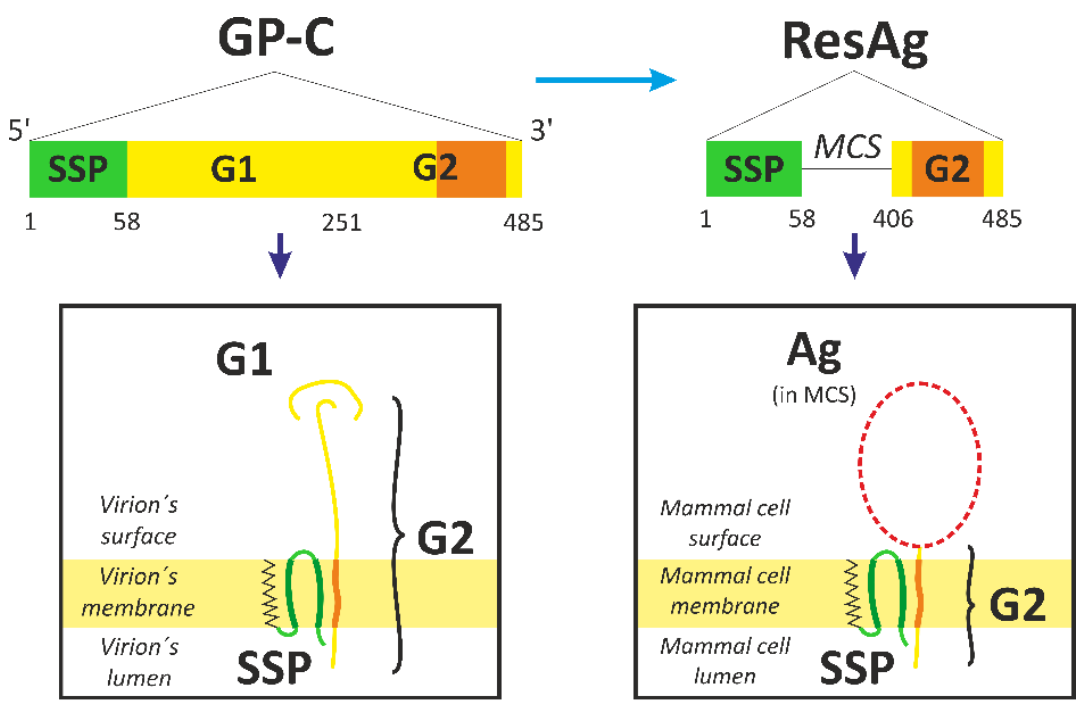

\section{$\operatorname{ResAg}(434 \mathrm{bps})$}

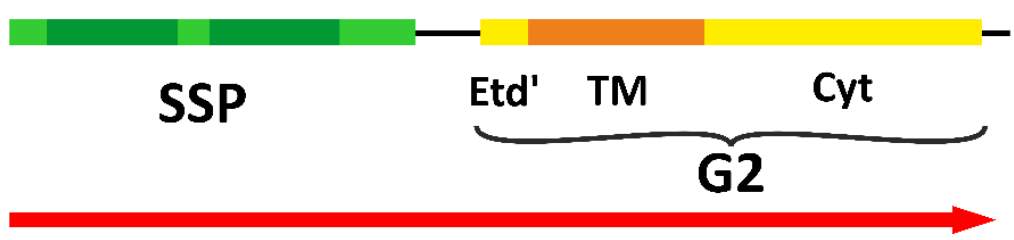

Figure 2. Genetic engineering of the expression vector pResAg. Top- Schematic representation of the GP-C sequence and the selection of its minimum elements, and the virion membrane as well as the membrane of a mammal cell expressing "ResAg"; Bottom- Details of the ResAg ORF (red arrow); GP-C: glycoprotein complex; SSP: stable signal peptide; G1: glycoprotein 1; G2: glycoprotein 2; MCS: multiple cloning site; Ag: antigen.

\subsection{Limitations of VLP-based vaccines}

As described above, VLPs can be considered antigen presentation systems that induce powerful and broad immune responses against several target antigens. VLPs are also biosecure and have immunogenicity advantages over the traditional approaches. There is a clear trend towards the use of VLPs as platforms for the development of many prophylactic vaccines. However, there are technical and practical considerations that may limit the application of VLP vaccines. First, due to its particulate nature, vaccines based on VLPs can only be effective against specific B or T cell epitopes that are highly conserved. Many pathogens undergo antigenic variation in response to host immune pressures during the course of infection and VLPs would not induce long-lasting immune responses against variant antigens. Secondly, considering immunological stress or the low rate of biodegradability, the possible toxicity of VLPs in the organism should not be ignored. Toxicity studies should be designed to maximize the detection of toxic effects resulting from the primary or secondary pharmacological action of VLP vaccines [49]. Finally, 
not all viruses can produce VLPs and some of those capable of generate VLPs cannot be used as vaccine candidates. Last but not least, pre-existing or maternal antibodies against VLPs can adversely affect immunogenicity, which would result in a failure vaccination [63].

\section{Discussion}

VLP technology is a rising field thanks to the areas of molecular biology and structural biology. VLPs of zoonotic viruses generated by structural elements of mature virions have received considerable attention during the last two decades. In fact, the data collected from the field suggest that VLPs are more effective than many other types of subunit vaccines against different infectious diseases. Recently, therapeutic vaccines based on VLPs have been developed with the aim of inducing humoral response against molecules that are involved in pathologies or chronic processes [63]. After these human vaccines based on VLPs were evaluated clinically, several important goals have been achieved: the reduction of blood pressure in hypertensive patients, the improvement of smoking abstinence after inoculation with a nicotine vaccine [64] and high antibody titers and cellular immune response against Plasmodium falciparum [65], . Also, to facilitate preliminary clinical trials, new non-clinical study programs have been developed [49]. The therapeutic vaccines can also be applied in the veterinary field to alleviate the suffering of animals with chronic diseases that cannot be sacrificed considering animal welfare.

Finally, numerous successful cases have been reported and new applications to VLPs continue to be described, being the VLPs-based vaccines the most promising field in preventive medicine during the 21st century.

Funding: This research was funded by the PICT 0880 granted by the Fondo para la Investigacion Cientifica y Tecnologica of the Agencia Nacional Cientifica y Tecnologica (FONCyT-ANPCyT), Republic of Argentina.

Conflicts of Interest: The authors declare no conflict of interest.

\section{References}

[1] World Health Organisation, 'Global Vaccine Action Plan', Vaccine, pp. 1-147, 2011.

[2] S. Plotkin, 'History of vaccination', Proc. Natl. Acad. Sci., vol. 111, no. 34, pp. 1228312287, Aug. 2014.

[3] S. A. Plotkin, 'Vaccines: The fourth century', Clin. Vaccine Immunol., vol. 16, no. 12, pp. 1709-1719, 2009.

[4] C. D. Rinaudo, J. L. Telford, R. Rappuoli, and K. L. Seib, 'Vaccinology in the genome era', J. Clin. Invest., vol. 119, no. 9, pp. 2515-2525, 2009.

[5] R. Rappuoli, 'Bridging the knowledge gaps in vaccine design.', Nat. Biotechnol., vol. 25, no. 12, pp. 1361-6, 2007.

[6] S. K. Parida and S. H. E. Kaufmann, 'Novel tuberculosis vaccines on the horizon', Curr. Opin. Immunol., vol. 22, no. 3, pp. 374-384, 2010.

[7] R. Rappuoli, 'Bridging the knowledge gaps in vaccine design.', Nat. Biotechnol., vol. 25, no. 12, pp. 1361-6, 2007.

[8] N. P. McCurley et al., 'HIV transmitted/founder vaccines elicit autologous tier 2 neutralizing antibodies for the CD4 binding site', PLOS ONE, vol. 12, no. 10, p. e0177863, Oct. 2017.

[9] F. Tohidi, S. M. Sadat, A. Bolhassani, R. Yaghoubi, and M. S. Larijani, 'Induction of a robust humoral response using HIV-1 VLPMPER-V3as a novel candidate vaccine in BALB/c mice', Curr. HIV Res., Mar. 2019. 
[10] M. L. Mbow, E. De Gregorio, N. M. Valiante, and R. Rappuoli, 'New adjuvants for human vaccines.', Curr. Opin. Immunol., vol. 22, no. 3, pp. 411-416, Jun. 2010.

[11] R. Capelli et al., 'BPSL1626: Reverse and Structural Vaccinology Reveal a Novel Candidate for Vaccine Design Against Burkholderia pseudomallei', Antibodies, vol. 7, no. 3, p. 26, Jul. 2018.

[12] R. H. M. Raeven, E. van Riet, H. D. Meiring, B. Metz, and G. F. A. Kersten, 'Systems vaccinology and big data in the vaccine development chain', Immunology, Oct. 2018.

[13] B. Capecchi et al., 'Neisseria meningitidis NadA is a new invasin which promotes bacterial adhesion to and penetration into human epithelial cells', Mol. Microbiol., vol. 55, no. 3, pp. 687-698, 2005.

[14] R. Rosini et al., 'Identification of novel genomic islands coding for antigenic pilus-like structures in Streptococcus agalactiae', Mol. Microbiol., vol. 61, no. 1, pp. 126-141, 2006.

[15] M. Pizza et al., 'Identification of Vaccine Candidates Against Serogroup B Meningococcus by Whole-Genome Sequencing', Science, vol. 287, no. 5459, pp. 1816-1820, 2000.

[16] D. Maione et al., 'Identification of a Universal Group B Streptococcus Vaccine by Multiple Genome Screen', vol. 309, no. 5731, pp. 148-150, 2006.

[17] D. G. Moriel et al., 'Identification of protective and broadly conserved vaccine antigens from the genome of extraintestinal pathogenic Escherichia coli.', Proc. Natl. Acad. Sci. U. S. A., vol. 107, no. 20, pp. 9072-7, 2010.

[18] A. D. M. E. Osterhaus, P. Koraka, and B. E. E. Martina, 'Bioinformatics in new generation flavivirus vaccines', J. Biomed. Biotechnol., vol. 2010, 2010.

[19] F. Cantini and L. Banci, 'Structural Knowledge for Molecular Optimization: The Cases of Metal-Mediated Protein-Protein Interactions and Structural Vaccinology: Structural Knowledge for Molecular Optimization: The Cases of Metal-Mediated Protein-Protein Interactions and Structural Vaccinology', Eur. J. Inorg. Chem., vol. 2018, no. 37, pp. 4108-4116, Oct. 2018.

[20] P. R. Dormitzer, G. Grandi, and R. Rappuoli, 'Structural vaccinology starts to deliver.', Nat. Rev. Microbiol., vol. 10, no. 12, pp. 807-13, 2012.

[21] A. F. Rodrigues, H. R. Soares, M. R. Guerreiro, P. M. Alves, and A. S. Coroadinha, 'Viral vaccines and their manufacturing cell substrates: New trends and designs in modern vaccinology', Biotechnol. J., vol. 10, no. 9, pp. 1329-1344, Sep. 2015.

[22] V. F. De La Cruz, A. A. Lal, and T. F. McCutchan, 'Immunogenicity and epitope mapping of foreign sequences via genetically engineered filamentous phage', J. Biol. Chem., vol. 263, no. 9, pp. 4318-4322, 1988.

[23] N. R. Burns et al., 'Symmetry, flexibility and permeability in the structure of yeast retrotransposon virus-like particles.', EMBO J., vol. 11, no. 3, pp. 1155-64, 1992.

[24] A. S. Belyaev and P. Roy, 'Presentation of hepatitis B virus preS2 epitope on bluetongue virus core-like particles.', Virology, vol. 190, no. 2, pp. 840-844, Oct. 1992.

[25] C. S. Brown, S. Welling-Wester, M. Feijlbrief, J. W. Van Lent, and W. J. Spaan, Chimeric parvovirus B19 capsids for the presentation of foreign epitopes., vol. 198. 1994. 
[26] D. J. Hwang, I. M. Roberts, and T. M. Wilson, 'Assembly of tobacco mosaic virus and TMV-like pseudovirus particles in Escherichia coli.', Arch. Virol. Suppl., vol. 9, pp. 543-558, 1994.

[27] J. Paavonen et al., 'Efficacy of a prophylactic adjuvanted bivalent L1 virus-like-particle vaccine against infection with human papillomavirus types 16 and 18 in young women: an interim analysis of a phase III double-blind, randomised controlled trial', The Lancet, vol. 369, no. 9580, pp. 2161-2170, Feb. 2017.

[28] M. O. Mohsen, L. Zha, G. Cabral-Miranda, and M. F. Bachmann, 'Major findings and recent advances in virus-like particle (VLP)-based vaccines', Semin. Immunol., vol. 34, pp. 123-132, Dec. 2017.

[29] E. Blanco, Structure and Physics of Viruses, vol. 68. Dordrecht: Springer Netherlands, 2013.

[30] D. Kim et al., 'Induction of type I interferon secretion through recombinant Newcastle disease virus expressing measles virus hemagglutinin stimulates antibody secretion in the presence of maternal antibodies.', J. Virol., vol. 85, no. 1, pp. 200-7, Enero 2011.

[31] Y.-L. Lin et al., 'Enterovirus-71 Virus-Like Particles Induce the Activation and Maturation of Human Monocyte-Derived Dendritic Cells through TLR4 Signaling.', PloS One, vol. 9, no. 10, p. e111496, 2014.

[32] R. Minkner et al., 'Purification of human papillomavirus-like particles expressed in silkworm using a Bombyx mori nucleopolyhedrovirus bacmid expression system', $J$. Chromatogr. B, vol. 1096, pp. 39-47, Oct. 2018.

[33] A. Rampoldi et al., 'Targeted Elimination of Tumorigenic Human Pluripotent Stem Cells Using Suicide-Inducing Virus-like Particles', ACS Chem. Biol., vol. 13, no. 8, pp. 2329-2338, Aug. 2018.

[34] T. M. Kündig, L. Klimek, P. Schendzielorz, W. A. Renner, G. Senti, and M. F. Bachmann, 'Is The Allergen Really Needed in Allergy Immunotherapy?', Curr. Treat. Options Allergy, vol. 2, no. 1, pp. 72-82, 2015.

[35] H. Daniell, V. Rai, and Y. Xiao, 'Cold chain and virus-free oral polio booster vaccine made in lettuce chloroplasts confers protection against all three poliovirus serotypes', Plant Biotechnol. J., Jan. 2019.

[36] N. Kushnir, S. J. Streatfield, and V. Yusibov, 'Virus-like particles as a highly efficient vaccine platform: Diversity of targets and production systems and advances in clinical development', Vaccine, vol. 31, no. 1, pp. 58-83, 2012.

[37] C. Yuste-Calvo, I. González-Gamboa, L. F. Pacios, F. Sánchez, and F. Ponz, 'Structure-Based Multifunctionalization of Flexuous Elongated Viral Nanoparticles', ACS Omega, vol. 4, no. 3, pp. 5019-5028, Mar. 2019.

[38] F. Liu, S. Ge, L. Li, X. Wu, Z. Liu, and Z. Wang, 'Virus-like particles: Potential veterinary vaccine immunogens', Res. Vet. Sci., vol. 93, no. 2, pp. 553-559, 2012.

[39] A. Zeltins, 'Construction and characterization of virus-like particles: A review', Mol. Biotechnol., vol. 53, no. 1, pp. 92-107, 2013.

[40] P. Roy and R. Noad, 'Virus-like particles as a vaccine delivery system: myths and facts', Adv Exp Med Biol, vol. 655, pp. 145-158, 2009. 
[41] A. Palladini et al., 'Virus-like particle display of HER2 induces potent anti-cancer responses', OncoImmunology, vol. 7, no. 3, p. e1408749, Mar. 2018.

[42] T. Fifis et al., 'Size-Dependent Immunogenicity: Therapeutic and Protective Properties of Nano-Vaccines against Tumors', J. Immunol., vol. 173, no. 5, pp. 3148-3154, 2004.

[43] A. Gamvrellis, D. Leong, J. C. Hanley, S. D. Xiang, P. Mottram, and M. Plebanski, 'Vaccines that facilitate antigen entry into dendritic cells', Immunol. Cell Biol., vol. 82, no. 5, pp. 506-516, 2004.

[44] A. S. Beignon et al., 'Endocytosis of HIV-1 activates plasmacytoid dendritic cells via Toll-like receptor-viral RNA interactions', J. Clin. Invest., vol. 115, no. 11, pp. 32653275, 2005.

[45] J. Zeng, P. Fournier, and V. Schirrmacher, 'Stimulation of human natural interferon-?? response via paramyxovirus hemagglutinin lectin-cell interaction', J. Mol. Med., vol. 80, no. 7, pp. 443-451, 2002.

[46] I. Frank et al., 'Infectious and whole inactivated simian immunodeficiency viruses interact similarly with primate dendritic cells (DCs): differential intracellular fate of virions in mature and immature DCs.', J. Virol., vol. 76, no. 6, pp. 2936-51, 2002.

[47] P. Lenz et al., 'Papillomavirus-like particles induce acute activation of dendritic cells', J Immunol, vol. 166, no. 9, pp. 5346-5355, 2001.

[48] M. Ostrowski, M. Vermeulen, O. Zabal, J. R. Geffner, a M. Sadir, and O. J. Lopez, 'Impairment of thymus-dependent responses by murine dendritic cells infected with foot-and-mouth disease virus', J Immunol, vol. 175, no. 6, pp. 3971-3979, 2005.

[49] M. F. Bachmann and G. T. Jennings, 'Vaccine delivery: a matter of size, geometry, kinetics and molecular patterns.', Nat. Rev. Immunol., vol. 10, no. 11, pp. 787-96, Nov. 2010.

[50] K. Madalinski, S. P. E. Sylvan, U. Hellstr??m, J. Mikolajewicz, E. Zembrzuska-Sadkowska, and E. Piontek, 'Antibody responses to preS components after immunization of children with low doses of BioHepB', Vaccine, vol. 20, no. 1-2, pp. 92-97, 2001.

[51] C. Grundner, T. Mirzabekov, J. Sodroski, and R. Wyatt, 'Solid-phase proteoliposomes containing human immunodeficiency virus envelope glycoproteins.', J. Virol., vol. 76, no. 7, pp. 3511-21, 2002.

[52] L. X. Doan, M. Li, C. Chen, and Q. Yao, 'Virus-like particles as HIV-1 vaccines.', Rev. Med. Virol., vol. 15, no. 2, pp. 75-88, 2005.

[53] G. B. McGaughey et al., 'Progress towards the development of a HIV-1 gp41-directed vaccine.', Curr. HIV Res., vol. 2, no. 2, pp. 193-204, Abril 2004.

[54] S. T. Agnandji, B. Lell, S. Solmeheim Soulanoudjingar, and J. F. Fernandes, 'First Results of Phase 3 Trial of RTS,S/AS01 Malaria Vaccine in African Children', N. Engl. J. Med., vol. 362, no. 20, pp. 1863-1875, 2011.

[55] S. Matić, R. Rinaldi, V. Masenga, and E. Noris, 'Efficient production of chimeric Human papillomavirus $16 \mathrm{~L} 1$ protein bearing the M2e influenza epitope in Nicotiana benthamiana plants', BMC Biotechnol., vol. 11, no. 1, p. 106, 2011. 
[56] X. Gao et al., 'Enhanced Influenza VLP vaccines comprising matrix-2 ectodomain and nucleoprotein epitopes protects mice from lethal challenge', Antiviral Res., vol. 98, no. 1, pp. 4-11, 2013.

[57] R. M. Jones et al., 'A plant-produced Pfs25 VLP malaria vaccine candidate induces persistent transmission blocking antibodies against plasmodium falciparum in immunized mice', PLoS ONE, vol. 8, no. 11, 2013.

[58] C. Dalba, B. Bellier, N. Kasahara, and D. Klatzmann, 'Replication-competent vectors and empty virus-like particles: new retroviral vector designs for cancer gene therapy or vaccines.', Mol. Ther. J. Am. Soc. Gene Ther., vol. 15, no. 3, pp. 457-466, 2007.

[59] B. Bellier and D. Klatzmann, 'Virus-like particle-based vaccines against hepatitis C virus infection.', Expert Rev. Vaccines, vol. 12, no. 2, pp. 143-54, 2013.

[60] C. S. Borio et al., 'Antigen vehiculization particles based on the Z protein of Junin virus.', BMC Biotechnol., vol. 12, p. 80, Enero 2012.

[61] V. A. Mareze et al., 'Tests in mice of a dengue vaccine candidate made of chimeric Junin virus-like particles and conserved dengue virus envelope sequences', Appl. Microbiol. Biotechnol., vol. 100, no. 1, pp. 125-133, Jan. 2016.

[62] De Ganzó, Desarrollo de una plataforma de presentación y vehiculización de antígenos basada en VLPs derivadas del virus Junín. Bernal, Buenos Aires, Argentina: UNQ, 2017.

[63] B. Chackerian, 'Virus-like particles: flexible platforms for vaccine development.', Expert Rev. Vaccines, vol. 6, no. 3, pp. 381-90, Jun. 2007.

[64] J. Cornuz et al., 'A Vaccine against Nicotine for Smoking Cessation: A Randomized Controlled Trial', PLoS ONE, vol. 3, no. 6, p. e2547, Jun. 2008.

[65] G. A. Oliveira et al., 'Safety and Enhanced Immunogenicity of a Hepatitis B Core Particle Plasmodium falciparum Malaria Vaccine Formulated in Adjuvant Montanide ISA 720 in a Phase I Trial', Infect. Immun., vol. 73, no. 6, pp. 3587-3597, Jun. 2005. 